\title{
Blow-up criteria for the 3D Bénard system in Besov spaces
}

Liangliang $\mathrm{Ma}^{1}$ and Lei Zhang ${ }^{2,3,4^{*}}$

\author{
"Correspondence: \\ scuzhanglei@scu.edu.cn; \\ zhanglei@scu.edu.cn \\ ${ }^{2}$ College of Computer Science, \\ Sichuan University, Chengdu, P.R. \\ China \\ ${ }^{3}$ State Key Laboratory of Plateau \\ Ecology and Agriculture, \\ Department of Computer \\ Technology and Applications, \\ Qinghai University, Xining, P.R. China \\ Full list of author information is \\ available at the end of the article
}

\begin{abstract}
This paper studies the regularity criteria of weak solutions for Bénard fluid flows in three dimensions. When the velocity field and the temperature belong to some critical Besov spaces on $(0, T)$, then the weak solution $(u, \theta)$ can be extended smoothly beyond $T$.
\end{abstract}

MSC: $75 B 03 ; 35 B 45 ; 35 B 65$

Keywords: Bénard system; Regularity criteria; Besov spaces; Weak solution; Global regularity

\section{Introduction}

In this paper, we consider the following three-dimensional (3D) Bénard system:

$$
\begin{aligned}
& \partial_{t} u+(u \cdot \nabla) u+\nabla \pi=\mu \Delta u+\theta e_{3}, \\
& \partial_{t} \theta+(u \cdot \nabla) \theta=\kappa \Delta \theta+u \cdot e_{3}, \\
& \nabla \cdot u=0, \quad \text { in } \mathbb{R}^{3} \times(0, \infty), \\
& u(0)=u_{0}, \quad \theta(0)=\theta_{0}, \quad \text { in } \mathbb{R}^{3},
\end{aligned}
$$

where $u=u(x, t) \in \mathbb{R}^{3}, \theta=\theta(x, t) \in \mathbb{R}$, and $\pi=\pi(x, t) \in \mathbb{R}$ represent the unknown velocity field, the temperature, and the pressure, respectively, the nonnegative constants $\mu$ and $\kappa$ are the coefficients of dissipation and thermal diffusivity, and $e_{3}=(0,0,1)^{T}$ denotes the vertical unit vector; the forcing term $\theta e_{3}$ in the momentum equation (1.1) describes the action of the buoyancy force on fluid motion, and $u \cdot e_{3}$ models the Rayleigh-Bénard convection in a heated inviscid fluid. We prescribe the initial data to satisfy the condition

$$
\nabla \cdot u_{0}=0
$$

The Bénard system describes the Rayleigh-Bénard convective motion in a heated 3D inviscid incompressible fluid under thermal effects (see, e.g., [1, 4, 8, 9, 21, 26]). We can easily check that if we ignore the Rayleigh-Bénard convection term $u \cdot e_{3}$, then system (1.1)-(1.4) becomes the corresponding Boussinesq system, which describes the evolution of the velocity field $u$ for a $3 \mathrm{D}$ incompressible fluid moving under the gravity and the earth rotation, which come from atmospheric or oceanographic turbulence, where rotation and

(c) The Author(s) 2019. This article is distributed under the terms of the Creative Commons Attribution 4.0 International License (http://creativecommons.org/licenses/by/4.0/), which permits unrestricted use, distribution, and reproduction in any medium, provided you give appropriate credit to the original author(s) and the source, provide a link to the Creative Commons license, and indicate if changes were made. 
stratification play an important role (see, e.g., $[3,6,7,22,25])$. In fact, the Boussinesq system is usually referred to as the Bénard convection problem.

Recently, the Bénard system has attracted considerable attention due to their physical applications and mathematical significance. Let us say some words about system (1.1)(1.4). Nakamura [24] established the smoothness and the time analyticity of strong solutions to the magnetic Bénard system in $\mathbb{R}^{n}, n=2,3$. Ma and Zhang [17] considered the global weak solution of the 2D Bénard system with partial dissipation and established some regularity criteria for the corresponding Bénard system. Later, they generalized and extended these results in [13]. It is currently unknown whether the solutions of the 3D magnetic Bénard system is globally regular (in time). Ma [14] dealt with the Cauchy problem to the 3D system of incompressible magnetic Bénard fluids and proved that as the initial data satisfy $\left\|u_{0}\right\|_{H^{1}\left(\mathbb{R}^{3}\right)}^{2}+\left\|\theta_{0}\right\|_{H^{1}\left(\mathbb{R}^{3}\right)}^{2}+\left\|b_{0}\right\|_{H^{1}\left(\mathbb{R}^{3}\right)}^{2} \leq \varepsilon$, where $\varepsilon$ is a suitably small positive number, the $3 \mathrm{D}$ magnetic Bénard system with mixed partial dissipation, magnetic diffusion, and thermal diffusivity admits a global smooth solution. Ma [15] investigated the blow-up criteria of strong solutions and regularity criterion of weak solutions for the magnetic Bénard system in $\mathbb{R}^{3}$ in a sense of scaling invariant by employing a different decomposition for nonlinear terms. More precisely, the strong solution $(u, \theta, b)$ of the magnetic Bénard system is proved to be smooth on $(0, T]$, provided that the velocity field $u$ satisfies

$$
u \in L^{\frac{2}{1-r}}\left(0, T ; \dot{\mathbb{X}}_{r}\left(\mathbb{R}^{3}\right)\right) \quad \text { with } 0 \leq r<1
$$

or the gradient field of velocity $\nabla u$ satisfies

$$
\nabla u \in L^{\frac{2}{2-\beta}}\left(0, T ; \dot{\mathbb{X}}_{\beta}\left(\mathbb{R}^{3}\right)\right) \quad \text { with } 0 \leq \beta \leq 1 .
$$

Moreover, he proved that if

$$
u \in L^{\infty}\left(0, T ; \dot{\mathbb{X}}_{1}\left(\mathbb{R}^{3}\right)\right) \quad \text { and } \quad\|u\|_{L^{\infty}\left(0, T ; \dot{\mathbb{X}}_{1}\left(\mathbb{R}^{3}\right)\right)}<\varepsilon
$$

where $\varepsilon>0$ is a suitable small constant, then the strong solution $(u, \theta, b)$ of magnetic Bénard system can also be extended beyond $t=T$. Finally, he showed that if some partial derivatives of the velocity components, magnetic components, and temperature components (i.e., $\tilde{\nabla} \tilde{u}, \tilde{\nabla} \theta$, and $\tilde{\nabla} \tilde{b})$ belong to the multiplier space, then the solution $(u, \theta, b)$ is in fact smooth on $(0, T)$. Very recently, Ma [16] studied the global regularity for the $2 \frac{1}{2} \mathrm{D}$ magnetic Bénard system with mixed partial viscosity. More precisely, he showed not only the global regularity for the $2 \frac{1}{2} \mathrm{D}$ magnetic Bénard system with zero thermal diffusivity by a well-known property of the Hardy space and BMO, but also obtained the global regularity for the $2 \frac{1}{2} \mathrm{D}$ magnetic Bénard system with zero thermal diffusivity and horizontal magnetic diffusion as well as vertical magnetic diffusion resorting to the method of the local-in-time analysis. Ma and Zhang [18] obtained a blow-up criterion of smooth solutions to the magnetic Bénard system without zero magnetic diffusion and thermal diffusivity in $\mathbb{R}^{2}$, derived a blow-up criterion of smooth solutions to the magnetic Bénard system without dissipation in $\mathbb{R}^{n}(n=2,3)$, and in what follows, they improved this result. In [19], they studied the Cauchy problem for the $2 \frac{1}{2} \mathrm{D}$ incompressible magnetic system with partial viscosity and derived some blow-up criteria of smooth solutions. In [20], the 
authors concentrated on the global regularity of classical solution to the $2 \frac{1}{2} \mathrm{D}$ magnetic Bénard system with partial dissipation, magnetic diffusion, and thermal diffusivity (i.e., horizontal dissipation, horizontal magnetic diffusion, and horziontal thermal diffusivity; vertical dissipation, vertical magnetic diffusion, and vertical thermal diffusivity).

Here it is worth particularly mentioning some regularity criteria for the fluid dynamic systems. Lin and Du [12] gave some global regularity criteria for 3D incompressible magnetohydrodynamics (MHD) equations. Mechdene et al. [23] established a sufficient condition for the regularity criterion of the Boussinesq equation in terms of the derivative of the pressure in one direction. Zhang [28] considered the 3D Hall-magnetohydrodynamic system, and showed that if the velocity and the magnetic fields belong to some critical Besov spaces on $(0, T)$, then the solution can be extended smoothly beyond $T$. Xu [27] considered the 3D magneto-micropolar fluid equations in Besov spaces.

Motivated by these results on the regularity criteria of fluid dynamics system, our aim in this study is to investigate the regularity criteria for the 3D Bénard system. To aid the introduction of our main results, let us first recall the definition of the first-time blow-up time (see [28]).

Definition 1.1 A number $T^{*}$ is called the first-time blow-up time if it satisfies the following conditions:

$$
\|(u, \theta)\|_{H^{r}}(t)<\infty \text { for all } t<T^{*},
$$

and

$$
\limsup _{t \nearrow T^{*}}\|(u, \theta)\|_{H^{r}}(t)=\infty
$$

where $r \geq 3$ is an integer, and $(u, \theta)$ is a solution to (1.1)-(1.4).

With the use of the notations described in Sect. 2, our main results now read as follows.

Theorem 1.1 Let $\left(u_{0}, \theta_{0}\right) \in H^{3}\left(\mathbb{R}^{3}\right)$ with $\nabla \cdot u_{0}=0$. If the velocity field $u$ and the gradient of the temperature $\nabla \theta$ satisfy

$$
\|u\|_{L^{1-s}\left(0, T^{*} ; \dot{B}_{\infty}^{-s}, \infty\right)}+\|\nabla \theta\|_{L^{2-\gamma}\left(0, T^{*} ; \dot{B}_{\infty, \infty}^{-\gamma}\right)}=\infty, \quad-1<s<1,0<\gamma<2,
$$

then for the first-time blow-up time $T^{*}<\infty$ of the solution $(u, \theta)$ to (1.1)-(1.4), we have that

$$
\underset{t \nearrow T^{*}}{\limsup }\|(u, \theta)\|_{H^{3}}^{2}(t)=\infty
$$

Using the Bernstein inequality (see Lemma 2.1), we easily obtain that $\nabla: \dot{B}_{p, q}^{r} \rightarrow \dot{B}_{p, q}^{r-1}$ is an isometry, and we can deduce from Theorem 1.1 the following:

Corollary 1.2 Let $\left(u_{0}, \theta_{0}\right) \in H^{3}\left(\mathbb{R}^{3}\right)$ with $\nabla \cdot u_{0}=0$. If the gradient field of the velocity $\nabla u$ and the gradient of the temperature $\nabla \theta$ satisfy

$$
\|\nabla u\|_{L^{2-s}\left(0, T^{*} ; \dot{B}_{\infty, \infty}^{-s}\right)}+\|\nabla \theta\|_{L^{\frac{2}{2-\gamma}}\left(0, T^{*} ; \dot{B}_{\infty, \infty}^{-\gamma}\right)}=\infty, \quad 0<s<2,0<\gamma<2,
$$


then for the first-time blow-up time $T^{*}<\infty$ of the solution $(u, \theta)$ to (1.1)-(1.4), we have that

$$
\limsup _{t \nearrow T^{*}}\|(u, \theta)\|_{H^{3}}^{2}(t)=\infty
$$

Because of the boundedness of the Riesz transformation in $\dot{B}_{p, q}^{r}\left(\mathbb{R}^{3}\right)$, we may further replace $\nabla u$ in Corollary 1.2 by the vorticity $\omega=\nabla \times u$ and obtain the following result.

Corollary 1.3 Let $\left(u_{0}, \theta_{0}\right) \in H^{3}\left(\mathbb{R}^{3}\right)$ with $\nabla \cdot u_{0}=0$. If the vorticity $\omega=\nabla \times u$ and the gradient of the temperature $\nabla \theta$ satisfy

$$
\|\omega\|_{L^{2-s}\left(0, T^{*} ; \dot{B}_{\infty, \infty}^{-s}\right)}+\|\nabla \theta\|_{L^{2-\gamma}\left(0, T^{*} ; \dot{B}_{\infty, \infty}^{-\gamma}\right)}=\infty, \quad 0<s<2,0<\gamma<2
$$

then for the first-time blow-up time $T^{*}<\infty$ of the solution $(u, \theta)$ to (1.1)-(1.4), we have that

$$
\limsup _{t \nearrow T^{*}}\|(u, \theta)\|_{H^{3}}^{2}(t)=\infty
$$

Theorem 1.4 Let $\left(u_{0}, \theta_{0}\right) \in H^{3}\left(\mathbb{R}^{3}\right)$ with $\nabla \cdot u_{0}=0$. If the velocity field $u$ and the temperature $\theta$ satisfy

$$
\|u\|_{L^{1-s}\left(0, T^{*} ; \dot{B}_{\infty, \infty}^{-s}\right)}+\|\theta\|_{L^{1-\gamma}\left(0, T^{*} ; \dot{B}_{\infty, \infty}^{-\gamma}\right)}=\infty, \quad-1<s<1,-1<\gamma<1,
$$

then for the first-time blow-up time $T^{*}<\infty$ of the solution $(u, \theta)$ to (1.1)-(1.4), we have that

$$
\limsup _{t \nearrow T^{*}}\|(u, \theta)\|_{H^{3}}^{2}(t)=\infty
$$

As an application of these results, we can easily deduce the following results.

Corollary 1.5 Let $\left(u_{0}, \theta_{0}\right) \in H^{3}\left(\mathbb{R}^{3}\right)$ with $\nabla \cdot u_{0}=0$. If the gradient of the velocity field $\nabla u$ and the temperature $\theta$ satisfy

$$
\|\nabla u\|_{L^{2-s}\left(0, T^{*} ; \dot{B}_{\infty, \infty}^{-s}\right)}+\|\theta\|_{L^{\frac{2}{1-\gamma}}\left(0, T^{*} ; \dot{B}_{\infty, \infty}^{-\gamma}\right)}=\infty, \quad 0<s<2,-1<\gamma<1,
$$

then for the first-time blow-up time $T^{*}<\infty$ of the solution $(u, \theta)$ to (1.1)-(1.4), we have that

$$
\limsup _{t \nearrow T^{*}}\|(u, \theta)\|_{H^{3}}^{2}(t)=\infty
$$

Corollary 1.6 Let $\left(u_{0}, \theta_{0}\right) \in H^{3}\left(\mathbb{R}^{3}\right)$ with $\nabla \cdot u_{0}=0$. If the vorticity $\omega=\nabla \times u$ and the temperature $\theta$ satisfy

$$
\|\omega\|_{L^{2-s}\left(0, T^{*} ; \dot{B}_{\infty, \infty}^{-s}\right)}+\|\theta\|_{L^{1-\gamma}\left(0, T^{*} ; \dot{B}_{\infty, \infty}^{-\gamma}\right)}=\infty, \quad 0<s<2,-1<\gamma<1,
$$

then for the first-time blow-up time $T^{*}<\infty$ of the solution $(u, \theta)$ to $(1.1)-(1.4)$, wehave that

$$
\limsup _{t \nearrow T^{*}}\|(u, \theta)\|_{H^{3}}^{2}(t)=\infty
$$




\section{Preliminaries}

Throughout this paper, the letter $C$ denotes various positive and finite constants that may vary from line to line. We will use the notation $A \lesssim B$ to denote the relation $A \leq C B$ and the notation $A \approx B$ to denote the relations $A \lesssim B$ and $B \lesssim A$. For reader's convenience, we first recall some basic facts on Littlewood-Paley theory; we refer to [5] for more detail.

Let $\mathcal{S}\left(\mathbb{R}^{3}\right)$ be the Schwartz class of rapidly decreasing functions. The Fourier transform $\mathcal{F} f=\hat{f}$ of $f \in \mathcal{S}\left(\mathbb{R}^{3}\right)$ is defined by

$$
\mathcal{F} f(\xi)=\hat{f}(\xi)=(2 \pi)^{-\frac{3}{2}} \int_{\mathbb{R}^{3}} e^{-i x \cdot \xi} f(x) d x .
$$

Choose two nonnegative radial functions $\chi, \varphi \in \mathcal{S}\left(\mathbb{R}^{3}\right)$ supported in $\mathfrak{B}=\left\{\xi \in \mathbb{R}^{3}:|\xi| \leq\right.$ $\left.\frac{4}{3}\right\}$ and $\mathcal{C}=\left\{\xi \in \mathbb{R}^{3}: \frac{3}{4} \leq|\xi| \leq \frac{8}{3}\right\}$, respectively, such that

$$
\chi(\xi)+\sum_{j \geq 0} \varphi\left(2^{-j} \xi\right)=1, \quad \xi \in \mathbb{R}^{3} .
$$

Set $\varphi_{j}(\xi)=\varphi\left(2^{-j} \xi\right)$ and let $h=\mathcal{F}^{-1} \varphi$ and $\tilde{h}=\mathcal{F}^{-1} \chi$. Then we define the frequency localization operators as follows:

$$
\begin{aligned}
& \Delta_{j} f=\varphi\left(2^{-j} D\right) f=2^{3 j} \int_{\mathbb{R}^{3}} h\left(2^{j} y\right) f(x-y) d y \quad \text { for } j \geq 0, \\
& S_{j} f=\chi\left(2^{-j} D\right) f=\sum_{-1 \leq k \leq j-1} \Delta_{k} f=2^{3 j} \int_{\mathbb{R}^{3}} \tilde{h}\left(2^{j} y\right) f(x-y) d y, \\
& \Delta_{-1} f=S_{0} f, \quad \Delta_{j} f=0 \quad \text { for } j \leq 2 .
\end{aligned}
$$

Formally, $\Delta_{j}=S_{j+1}-S_{j}$ is a frequency projection into the annulus $\left\{|\xi| \approx 2^{j}\right\}$, whereas $S_{j}$ is a frequency projection into the ball $\left\{|\xi| \lesssim 2^{j}\right\}$ for $j \in \mathbb{Z}$. We can easily verify that, with the our choice of $\varphi$,

$$
\Delta_{j} \Delta_{k} f \equiv 0 \quad \text { if }|j-k| \geq 2,
$$

and

$$
\Delta_{j}\left(S_{k-1} f \Delta_{k} f\right) \equiv 0 \quad \text { if }|j-k| \geq 5
$$

We now introduce the following definition of a homogeneous Besov space by means of Littlewood-Paley projection $\Delta_{j}$ and $S_{j}$.

Definition 2.1 Let $r \in \mathbb{R}$ and $1 \leq p, q \leq \infty$. The homogeneous Besov space $\dot{B}_{p, q}^{r}\left(\mathbb{R}^{3}\right)$ is defined by the full dyadic decomposition

$$
\dot{B}_{p, q}^{r}=\left\{f \in \mathcal{Z}^{\prime}\left(\mathbb{R}^{3}\right) ;\|f\|_{\dot{B}_{p, q}^{r}}<\infty\right\},
$$

where

$$
\|f\|_{\dot{B}_{p, q}^{r}}=\left\|\left\{2^{j r}\left\|\Delta_{f} f\right\|_{L^{p}}\right\}_{j=-\infty}^{\infty}\right\|_{\ell q} .
$$


Let us point out that $\mathcal{Z}^{\prime}\left(\mathbb{R}^{3}\right)$ is the dual space of

$$
\mathcal{Z}\left(\mathbb{R}^{3}\right)=\left\{f \in \mathcal{S}\left(\mathbb{R}^{3}\right) ; D^{\alpha} \widehat{f}(0)=0 \forall \alpha \in \mathbb{N}^{3}\right\}
$$

The following Bernstein inequality is due to $[5,11]$.

Lemma 2.1 For all $k \in \mathbb{N} \cup\{0\}, j \in \mathbb{Z}$, and $1 \leq p \leq q \leq \infty$, we have, for all $f \in \mathcal{S}\left(\mathbb{R}^{3}\right)$,

(i) $\sup _{|\alpha|=k}\left\|\nabla^{\alpha} \Delta_{j} f\right\|_{L^{q}} \leq C_{1} 2^{j k+3 j\left(\frac{1}{p}-\frac{1}{q}\right)}\left\|\Delta_{j} f\right\|_{L^{p}}$,

(ii) $\quad\left\|\Delta_{j} f\right\|_{L^{p}} \leq C_{2} 2^{-j k} \sup _{|\alpha|=k}\left\|\nabla^{\alpha} \Delta_{j} f\right\|_{L^{p}}$,

with positive constants $C_{1}$ and $C_{2}$ independent of $f$ and $j$.

We conclude this section by recalling the refined interpolation inequality relating the Lebesgue and Besov spaces (see [2]), which will be frequently used in the proof of Theorem 1.1.

Lemma 2.2 Let $1 \leq q<p<\infty$, and let $\alpha$ be a positive real number. There exists a constant C such that

$$
\|f\|_{L^{p}} \leq C\|f\|_{\dot{B}_{\infty, \infty}^{-\alpha}}^{1-\gamma}\|f\|_{\dot{B}_{q, q}^{\beta}}^{\gamma} \quad \text { with } \beta=\alpha\left(\frac{p}{q}-1\right) \text { and } \gamma=\frac{q}{p} \text {. }
$$

\section{Proof of Theorem 1.1}

In this section, we prove Theorem 1.1. We will complete the proof through three steps. First, we will get the basic energy inequality. Second, we will establish lower-order estimates of $u$ and $\theta$. Finally, we will obtain higher-order estimates of $u$ and $\theta$. To prove Theorem 1.1, we will apply a contradiction argument method. More explicitly, we need to show that if

$$
\|u\|_{L \frac{2}{1-s}\left(0, T^{*} ; \dot{B}_{\infty, \infty}^{-s}\right)}+\|\nabla \theta\|_{L^{2-\gamma}\left(0, T^{*} ; \dot{B}_{\infty, \infty}^{-\gamma}\right)}<\infty
$$

for some $-1<s<1$ and $0<\gamma<2$, then we could conduct uniform bounds for $\|(u, \theta)\|_{H^{m}}(t)$ for $0 \leq t<T^{*}, m \geq 3$. Then we give a proof of Theorem 1.1. First, we will establish the following basic energy estimate for system (1.1)-(1.4).

Lemma 3.1 Let $\left(u_{0}, \theta_{0}\right)$ satisfy the conditions of Theorem 1.1. Then for any corresponding solution $(u, \theta)$ of system (1.1)-(1.4), there exists a constant $C$ such that, for almost every $t \geq 0$,

$$
\|u(t)\|_{L^{2}}^{2}+\|\theta(t)\|_{L^{2}}^{2}+\mu \int_{0}^{t}\|\nabla u(\tau)\|_{L^{2}}^{2} d \tau+\kappa \int_{0}^{t}\|\nabla \theta(\tau)\|_{L^{2}}^{2} d \tau \leq C,
$$

where $C$ is a constant depending on time $t$ and the initial data. 
Proof Multiplying (1.1) and (1.2) by $u$ and $\theta$, respectively, integrating them over $\mathbb{R}^{3}$ with respect to $x$, and then adding the resulting equations, we get

$$
\frac{1}{2} \frac{d}{d t}\left(\|u(t)\|_{L^{2}}^{2}+\|\theta(t)\|_{L^{2}}^{2}\right)+\mu\|\nabla u\|_{L^{2}}^{2}+\kappa\|\nabla \theta\|_{L^{2}}^{2} \leq C\left(\|u\|_{L^{2}}^{2}+\|\theta\|_{L^{2}}^{2}\right) .
$$

Integration of $(3.1)$ with respect to time variable over $(0, t)$ directly implies the desired estimate (3.1).

Next, we will establish the global $H^{1}$-estimation, which plays an essential role in proving our main theorem.

Lemma 3.2 Let $\left(u_{0}, \theta_{0}\right)$ satisfy the conditions of Theorem 1.1. Then for any corresponding solution $(u, \theta)$ of the system (1.1)-(1.4), there exists a constant $C$ such that, for any $0 \leq t<$ $T^{*}$,

$$
\|\nabla u(t)\|_{L^{2}}^{2}+\|\nabla \theta(t)\|_{L^{2}}^{2}+\mu \int_{0}^{t}\|\Delta u(\tau)\|_{L^{2}}^{2} d \tau+\kappa \int_{0}^{t}\|\Delta \theta(\tau)\|_{L^{2}}^{2} d \tau \leq C<\infty,
$$

where $C$ is a constant depending on time $t$ and the initial data.

Proof Taking the $L^{2}$ inner product to Eqs. (1.1) and (1.2) with $-\Delta u$ and $-\Delta \theta$, respectively, and summing up the resultants, we obtain

$$
\begin{aligned}
\frac{1}{2} \frac{d}{d t}\left(\|\nabla u(t)\|_{L^{2}}^{2}+\|\nabla \theta(t)\|_{L^{2}}^{2}\right)+\mu\|\Delta u\|_{L^{2}}^{2}+\kappa\|\Delta \theta\|_{L^{2}}^{2} \\
=\int_{\mathbb{R}^{3}}[(u \cdot \nabla) u] \cdot \Delta u d x-\int_{\mathbb{R}^{3}} \theta e_{3} \cdot \Delta u d x \\
\quad+\int_{\mathbb{R}^{3}}[(u \cdot \nabla) \theta] \cdot \Delta \theta d x-\int_{\mathbb{R}^{3}}\left(u \cdot e_{3}\right) \cdot \Delta \theta d x \\
:=I+I I+I I I+I V .
\end{aligned}
$$

Using the incompressible condition, the Hölder inequality, the interpolation inequality, and Lemma 2.2, we estimate $I$ and $I I I$ as follows:

$$
\begin{aligned}
I & =-\sum_{i=1}^{3} \int_{\mathbb{R}^{3}}\left[\left(\partial_{i} u \cdot \nabla\right) u\right] \cdot \partial_{i} u d x \leq C\|\nabla u\|_{L^{3}}^{3} \\
& \leq C\|\nabla u\|_{\dot{B}_{\infty, \infty}^{-1-s}}\|\nabla u\|_{\dot{H}^{\frac{s+1}{2}}}^{2} \leq C\|u\|_{\dot{B}_{\infty, \infty}^{-s}}\|\nabla u\|_{L^{2}}^{1-s}\|\nabla u\|_{\dot{H}^{1}}^{1+s} \\
& \leq \frac{\mu}{4}\|\Delta u\|_{L^{2}}^{2}+C\|u\|_{\dot{B}_{\infty, \infty}^{-s}}^{\frac{2}{1-s}}\|\nabla u\|_{L^{2}}^{2}, \\
I I I & =-\sum_{i=1}^{3} \int_{\mathbb{R}^{3}}\left[\left(\partial_{i} u \cdot \nabla\right) \theta\right] \cdot \partial_{i} \theta d x \leq C \int_{\mathbb{R}^{3}}|\nabla u| \cdot|\nabla \theta|^{2} d x \\
& \leq C\|\nabla u\|_{L^{3}}\|\nabla \theta\|_{L^{3}}^{2} \leq C\|\nabla u\|_{L^{3}}^{3}+C\|\nabla \theta\|_{L^{3}}^{3} \\
& \leq C\|\nabla u\|_{\dot{B}_{\infty, \infty}^{-1-s}}\|\nabla u\|_{\dot{H}^{\frac{s+1}{2}}}^{2}+C\|\nabla \theta\|_{\dot{B}_{\infty, \infty}^{-\gamma}}\|\nabla \theta\|_{\dot{H}^{\frac{\gamma}{2}}}^{2}
\end{aligned}
$$




$$
\begin{aligned}
& \leq C\|u\|_{\dot{B}_{\infty, \infty}^{-s}}\|\nabla u\|_{L^{2}}^{1-s}\|\nabla u\|_{\dot{H}^{1}}^{1+s}+C\|\nabla \theta\|_{\dot{B}_{\infty, \infty}^{-\gamma}}\|\nabla \theta\|_{L^{2}}^{2-\gamma}\|\nabla \theta\|_{\dot{H}^{1}}^{\gamma} \\
& \leq \frac{\mu}{4}\|\Delta u\|_{L^{2}}^{2}+\frac{\kappa}{2}\|\Delta \theta\|_{L^{2}}^{2}+C\left(\|u\|_{\dot{B}_{\infty, \infty}^{-s}}^{\frac{2}{1-s}}+\|\nabla \theta\|_{\dot{B}_{\infty, \infty}^{-\gamma}}^{\frac{2}{2-\gamma}}\right)\left(\|\nabla u\|_{L^{2}}^{2}+\|\nabla \theta\|_{L^{2}}^{2}\right) .
\end{aligned}
$$

By the integration-by-parts formula and Hölder inequality we easily observe that

$$
I I+I V \leq C\left(\|\nabla u\|_{L^{2}}^{2}+\|\nabla \theta\|_{L^{2}}^{2}\right)
$$

Putting estimates $I-I V$ into (3.4) and absorbing the diffusive terms ensure that

$$
\begin{aligned}
& \frac{d}{d t}\left(\|\nabla u(t)\|_{L^{2}}^{2}+\|\nabla \theta(t)\|_{L^{2}}^{2}\right)+\mu\|\Delta u\|_{L^{2}}^{2}+\kappa\|\Delta \theta\|_{L^{2}}^{2} \\
& \quad \leq C\left(1+\|u\|_{\dot{B}_{\infty, \infty}^{-s}}^{\frac{2}{1-s}}+\|\nabla \theta\|_{\dot{B}_{\infty, \infty}^{-\gamma}}^{\frac{2}{2-\gamma}}\right) \cdot\left(\|\nabla u\|_{L^{2}}^{2}+\|\nabla \theta\|_{L^{2}}^{2}\right) .
\end{aligned}
$$

Thanks to the Gronwall inequality, we show that, for any $0 \leq t<T^{*}$,

$$
\begin{aligned}
& \|\nabla u(t)\|_{L^{2}}^{2}+\|\nabla \theta(t)\|_{L^{2}}^{2}+\mu \int_{0}^{t}\|\Delta u(\tau)\|_{L^{2}}^{2} d \tau+\kappa \int_{0}^{t}\|\Delta \theta(\tau)\|_{L^{2}}^{2} d \tau \\
& \quad \leq\left(\left\|\nabla u_{0}\right\|_{L^{2}}^{2}+\left\|\nabla \theta_{0}\right\|_{L^{2}}^{2}\right) \exp \left\{C T^{*}+\int_{0}^{T^{*}}\left(\|u(\tau)\|_{\dot{B}_{\infty, \infty}^{-s}}^{\frac{2}{1-s}}+\|\nabla \theta(\tau)\|_{\dot{B}_{\infty, \infty}^{-\gamma}}^{\frac{2}{2-\gamma}}\right) d \tau\right\} \\
& \quad \leq C<\infty .
\end{aligned}
$$

Therefore we finally conclude the proof of Lemma 3.2.

With the help of Lemma 3.2, we establish the global $H^{3}$-estimation.

Lemma 3.3 Let $\left(u_{0}, \theta_{0}\right)$ satisfies the conditions of Theorem 1.1. Then for any corresponding solution $(u, \theta)$ of system (1.1)-(1.4), there exists a constant $C$ such that, for any $0 \leq t<T^{*}$,

$$
\|u(t)\|_{\dot{H}^{3}}^{2}+\|\theta(t)\|_{\dot{H}^{3}}^{2}+\mu \int_{0}^{t}\|\nabla u(\tau)\|_{\dot{H}^{3}}^{2} d \tau+\kappa \int_{0}^{t}\|\nabla \theta(\tau)\|_{\dot{H}^{3}}^{2} d \tau \leq C<\infty
$$

where $C$ is a constant depending on time $t$ and the initial data.

Proof Let $\alpha=\left(\alpha_{1}, \alpha_{2}, \alpha_{3}\right)$ be a multiindex with $|\alpha|=\alpha_{1}+\alpha_{2}+\alpha_{3}=3$. Applying the operator $D^{\alpha}=\frac{\partial^{|\alpha|}}{\partial x_{1}^{\alpha_{1}} \partial x_{2}^{\alpha_{2}} \partial x_{3}^{\alpha_{3}}}$, where $0 \leq \alpha \leq 3$, to equations (1.1) and (1.2) and then taking the $L^{2}$ inner product of the resulting equations with $D^{\alpha} u$ and $D^{\alpha} \theta$, after adding them, we obtain that

$$
\begin{aligned}
\frac{1}{2} \frac{d}{d t}\left(\|u(t)\|_{\dot{H}^{3}}^{2}+\|\theta(t)\|_{\dot{H}^{3}}^{2}\right)+\mu\|\nabla u\|_{\dot{H}^{3}}^{2}+\kappa\|\nabla \theta\|_{\dot{H}^{3}}^{2} \\
=-\sum_{|\alpha|=3} \int_{\mathbb{R}^{3}} D^{\alpha}[(u \cdot \nabla) u] \cdot D^{\alpha} u d x+\sum_{|\alpha|=3} \int_{\mathbb{R}^{3}} D^{\alpha}\left(\theta e_{3}\right) \cdot D^{\alpha} u d x \\
\quad-\sum_{|\alpha|=3} \int_{\mathbb{R}^{3}} D^{\alpha}[(u \cdot \nabla) \theta] \cdot D^{\alpha} \theta d x+\sum_{|\alpha|=3} \int_{\mathbb{R}^{3}} D^{\alpha}\left(u \cdot e_{3}\right) \cdot D^{\alpha} \theta d x .
\end{aligned}
$$


Due to the divergence-free condition $\nabla \cdot u=0$, we can rewrite (3.11) as

$$
\begin{aligned}
\frac{1}{2} \frac{d}{d t}\left(\|u(t)\|_{\dot{H}^{3}}^{2}+\|\theta(t)\|_{\dot{H}^{3}}^{2}\right)+\mu\|\nabla u\|_{\dot{H}^{3}}^{2}+\kappa\|\nabla \theta\|_{\dot{H}^{3}}^{2} \\
=\sum_{i=1}^{3} \sum_{|\alpha|=3} \int_{\mathbb{R}^{3}} D^{\alpha}\left(u_{i} u\right) \cdot D^{\alpha} \partial_{i} u d x+\sum_{|\alpha|=3} \int_{\mathbb{R}^{3}} D^{\alpha}\left(\theta e_{3}\right) \cdot D^{\alpha} u d x \\
\quad+\sum_{i=1}^{3} \sum_{|\alpha|=3} \int_{\mathbb{R}^{3}} D^{\alpha}\left(u_{i} \theta\right) \cdot D^{\alpha} \partial_{i} \theta d x+\sum_{|\alpha|=3} \int_{\mathbb{R}^{3}} D^{\alpha}\left(u \cdot e_{3}\right) \cdot D^{\alpha} \theta d x \\
:=I_{1}+I I_{1}+I I I_{1}+I V_{1} .
\end{aligned}
$$

Before bounding terms in (3.12), we first recalling the following useful lemma (see [10]).

Lemma 3.4 If $r>0$ and $1<p<\infty$, then $W^{r, p} \cap L^{\infty}$ is an algebra. Moreover,

$$
\|f g\|_{W^{r, p}} \leq C\left(\|f\|_{L^{\infty}}\|g\|_{L^{p}}+\|f\|_{L^{p}}\|g\|_{L^{\infty}}\right)
$$

With Lemma 3.4 at our disposal, we are now ready to bound terms in (3.12) as follows:

$$
\begin{aligned}
I_{1} \leq & C\|u\|_{L^{\infty}}\|u\|_{H^{3}}\|\nabla u\|_{\dot{H}^{3}} \leq C\|u\|_{L^{\infty}}\left(\|u\|_{L^{2}}+\|u\|_{\dot{H}^{3}}\right)\|\nabla u\|_{\dot{H}^{3}} \\
& \leq \frac{\mu}{4}\|\nabla u\|_{\dot{H}^{3}}^{2}+C\|u\|_{L^{\infty}}^{2}\left(1+\|u\|_{\dot{H}^{3}}^{2}\right), \\
I I I_{1} & \leq C\|(u, \theta)\|_{L^{\infty}}\|(u, \theta)\|_{H^{3}}\|(\nabla u, \nabla \theta)\|_{\dot{H}^{3}} \\
& \leq C\|(u, \theta)\|_{L^{\infty}}\left(\|(u, \theta)\|_{L^{2}}+\|(u, \theta)\|_{\dot{H}^{3}}\right)\|(\nabla u, \nabla \theta)\|_{\dot{H}^{3}} \\
& \leq \frac{\mu}{4}\|\nabla u\|_{\dot{H}^{3}}^{2}+\frac{\kappa}{2}\|\nabla \theta\|_{\dot{H}^{3}}^{2}+C\|(u, \theta)\|_{L^{\infty}}^{2}\left(1+\|(u, \theta)\|_{\dot{H}^{3}}^{2}\right), \\
I I_{1} & +I V_{1} \leq C\left(\|u\|_{\dot{H}^{3}}^{2}+\|\theta\|_{\dot{H}^{3}}^{2}\right) .
\end{aligned}
$$

Substituting all the preceding estimates into (3.12) directly gives

$$
\begin{aligned}
& \frac{d}{d t}\left(\|u(t)\|_{\dot{H}^{3}}^{2}+\|\theta(t)\|_{\dot{H}^{3}}^{2}\right)+\mu\|\nabla u\|_{\dot{H}^{3}}^{2}+\kappa\|\nabla \theta\|_{\dot{H}^{3}}^{2} \\
& \quad \leq C\left(1+\|(u, \theta)\|_{L^{\infty}}^{2}\right)\left(1+\|(u, \theta)\|_{\dot{H}^{3}}^{2}\right) .
\end{aligned}
$$

A combination of this fact with the Gronwall inequality leads to the desired global bound in Lemma 3.3. We can also obtain

$$
\|(u, \theta)\|_{H^{m}}(t) \leq C, \quad t \in\left[0, T^{*}\right), m \geq 3 .
$$

This completes the proof of Theorem 1.1. 


\section{The proof of Theorem 1.4}

Due to the similarity to the proof in the previous section, the proof of Theorem 1.4 is much simpler. In fact, it is sufficient to change estimate (3.6) as follows:

$$
\begin{aligned}
I I I & \leq C\|\nabla u\|_{L^{3}}^{3}+C\|\nabla \theta\|_{L^{3}}^{3} \\
& \leq C\|\nabla u\|_{\dot{B}_{\infty, \infty}^{-1, s}}\|\nabla u\|_{\dot{H}^{\frac{s+1}{2}}}^{2}+C\|\nabla \theta\|_{\dot{B}_{\infty, \infty}^{-1-\gamma}}\|\nabla \theta\|_{\dot{H}^{\frac{\gamma+1}{2}}}^{2} \\
& \leq C\|u\|_{\dot{B}_{\infty, \infty}^{-s}}\|\nabla u\|_{L^{2}}^{1-s}\|\nabla u\|_{\dot{H}^{1}}^{1+s}+C\|\theta\|_{\dot{B}_{\infty, \infty}^{-\gamma}}\|\nabla \theta\|_{L^{2}}^{1-\gamma}\|\nabla \theta\|_{\dot{H}^{1}}^{1+\gamma} \\
& \leq \frac{\mu}{4}\|\Delta u\|_{L^{2}}^{2}+\frac{\kappa}{2}\|\Delta \theta\|_{L^{2}}^{2}+C\left(\|u\|_{\dot{B}_{\infty, \infty}^{-s}}^{\frac{2}{1-s}}+\|\theta\|_{\dot{B}_{\infty, \infty}^{-\gamma}}^{\frac{2}{1-\gamma}}\right)\left(\|\nabla u\|_{L^{2}}^{2}+\|\nabla \theta\|_{L^{2}}^{2}\right) .
\end{aligned}
$$

\section{Results and discussion}

In this paper, we consider the 3D Bénard system and investigate the regularity criteria for it. We prove that if the velocity field $u$ and the temperature $\theta$ belong to some critical Besov space, then the solution $(u, \theta)$ can be extended smoothly beyond $T$.

\section{Conclusions}

In this paper, we show that if the velocity field $u$ and the gradient of the temperature $\nabla \theta$ satisfy

$$
\|u\|_{L \frac{2}{1-s}\left(0, T^{*} ; \dot{B}_{\infty, \infty}^{-s}\right)}+\|\nabla \theta\|_{L^{2-\gamma}\left(0, T^{*} ; \dot{B}_{\infty, \infty}^{-\gamma}\right)}=\infty, \quad-1<s<1,0<\gamma<2,
$$

or the velocity field $u$ and the temperature $\theta$ satisfy

$$
\|u\|_{L^{1-s}\left(0, T^{*} ; \dot{B}_{\infty, \infty}^{-s}\right)}+\|\theta\|_{L^{1-\gamma}\left(0, T^{*} ; \dot{B}_{\infty, \infty}^{-\gamma}\right)}=\infty, \quad-1<s<1,-1<\gamma<1,
$$

then for the first-time blow-up time $T^{*}<\infty$ of the solution $(u, \theta)$, we have that

$$
\limsup _{t \nearrow T^{*}}\|(u, \theta)\|_{H^{3}}^{2}(t)=\infty
$$

\section{Acknowledgements}

This paper is partially supported by The National Natural Science Foundation of China (Nos. 61563044, 71702119, 61762074), National Natural Science Foundation of Qinghai Province (No. 2017-ZJ-902), Open Research Fund Program of State key Laboratory of Hydroscience and Engineering (No. sklhse-2017-A-05), and The Science and Technology Major Project of Qinghai Province Natural Science Foundation (No. 2015-SF-A4-3). The authors would like to express sincere gratitude to Professor Lili Du for guidance, constant encouragement, and providing an excellent research environment. The authors would also like to thank the referee for his/her pertinent comments and advice. We would like to declare on behalf of my coauthors that the work described was an original research not published previously and not under consideration for publication elsewhere, in whole or in part. Both authors have approved the manuscript.

\section{Competing interests}

The authors declare that they have no competing interests.

\section{Authors' contributions}

All authors read and approved the final manuscript.

\section{Author details}

${ }^{1}$ Geomathematics Key Laboratory of Sichuan Province, Chengdu University of Technology, Chengdu, P.R. China. ${ }^{2}$ College of Computer Science, Sichuan University, Chengdu, P.R. China. ${ }^{3}$ State Key Laboratory of Plateau Ecology and Agriculture, Department of Computer Technology and Applications, Qinghai University, Xining, P.R. China. ${ }^{4}$ Information Management Center, Sichuan University, Chengdu, P.R. China. 


\section{Publisher's Note}

Springer Nature remains neutral with regard to jurisdictional claims in published maps and institutional affiliations.

\section{Received: 12 February 2018 Accepted: 26 February 2019 Published online: 12 March 2019}

\section{References}

1. Ambrosetti, A., Prodi, G.: A Primer of Nonlinear Analysis. Cambridge Studies in Advanced Mathematics. Cambridge University Press, Cambridge (1995)

2. Bahouri, H., Chemin, J.Y., Danchin, R.: Fourier Analysis and Nonlinear Partial Differential Equations. Grundlehren Math. Wiss. (Fundamental Principles of Mathematical Sciences), vol. 343. Springer, Heidelberg (2011)

3. Cannon, J.R., DiBenedetto, E.: The Initial Value Problem for the Boussinesq Equations with Data in $L^{p}$, Approximation Methods for Navier-Stokes Problems. Lecture Notes in Math., vol. 771. Springer, Berlin (1980)

4. Chandrasekhar, S.: Hydrodynamic and Hydromagnetic Stability. Dover, New York (1981)

5. Chemin, J.-Y.: Perfect Incompressible Fluids, Translated from the 1995 French Original by Isabelle Gallagher and Dragos Iftimie. Oxford Lecture Series in Mathematics and Its Applications, vol. 14. Clarendon, New York (1998)

6. Chemin, J.-Y., Desjardins, B., Gallagher, I., Grenier, E.: Fluids with anisotropic viscosity. Math. Model. Numer. Anal. 34 315-335 (2000)

7. Chemin, J.-Y., Desjardins, B., Gallagher, I., Grenier, E.: Mathematical Geophysics: An Introduction to Rotating Fluids and to the Navier-Stokes Equations. Oxford University Press, London (2006)

8. Farhat, A., Jolly, M., Titi, E.: Continuous data assimilation for the 2D Bénard convection through velocity measurements alone. Physica D 33, 59-66 (2015)

9. Foias, C., Manley, O., Teman, R.: Attractors for the Bénard problem: existence and physical bounds on their fractal dimension. Nonlinear Anal. TMA 11, 939-967 (1987)

10. Kato, T., Ponce, G.: Commutator estimates and the Euler and Navier-Stokes equations. Commun. Pure Appl. Math. 41, 891-907 (1988)

11. Lemarié-Rieusset, P.G.: Recent Developments in the Navier-Stokes Problem. Chapman \& Hall, Boca Raton (2002)

12. Lin, H., Du, L.: Regularity criteria for incompressible magnetohydrodynamics equations in three dimensions. Nonlinearity 26, 219-239 (2013)

13. Ma, L.: Global regularity for the 2D magnetic Bénard fluid system with mixed partial viscosity. Comput. Math. Appl. 76 2148-2166 (2018)

14. Ma, L:: Global existence of smooth solutions for three-dimensional magnetic Bénard system with mixed partial dissipation, magnetic diffusion and thermal diffusivity. J. Math. Anal. Appl. 461, 1639-1652 (2018)

15. Ma, L.: Blow-up criteria and regularity criterion for the three-dimensional magnetic Bénard system in the multiplier space. Results Math. (2018). https://doi.org/10.1007/s00025-018-0860-3

16. Ma, L.: Global regularity results for the $2 \frac{1}{2} \mathrm{D}$ magnetic Bénard system with mixed partial viscosity. Appl. Anal. (2018). https://doi.org/10.1080/00036811.2017.1416103

17. Ma, L., Zhang, L.: Global existence of weak solution and regularity criteria for the $2 d$ Bénard system with partial dissipation. Bound. Value Probl. (2018). https://doi.org/10.1186/s13661-018-0988-9

18. Ma, L., Zhang, L.: Blow-up criteria for $2 \frac{1}{2} \mathrm{D}$ magnetic Bénard fluid system with partial viscosity. Appl. Math. Comput. (2018). https://doi.org/10.1016/j.amc.2018.10.008

19. Ma, L., Zhang, L.: Blow-up criteria for $2 \frac{1}{2}$ D magnetic Bénard fluid system with partial viscosity. Appl. Anal. (2018). https://doi.org/10.1080/00036811.2018.1529305

20. Ma, L., Zhang, L.: Regularity criteria for the two-and-half-dimensional magnetic Bénard system with partial dissipation, magnetic diffusion and thermal diffusivity. Bound. Value Probl. 2019, 30 (2019) https://doi.org/10.1186/s13661-019-1130-3

21. Ma, T., Wang, S.: Rayleigh-Bénard convection: dynamics and structure in the physical space. Commun. Math. Sci. 5, 553-574 (2007)

22. Majda, A., Grote, M.: Model dynamics and vertical collapse in decaying strongly stratified flows. Phys. Fluids 9 , 2932-2940 (1997)

23. Mechdene, M., Gala, S., Guo, Z., Ragusa, A.M.: Logarithmical regularity criterion of the three-dimensional Boussinesq equations in terms of the pressure. Z. Angew. Math. Phys. 67, 1-10 (2016)

24. Nakamura, M.: Regularity and analyticity of the solutions of the magnetic Bénard problem. Adv. Math. Sci. Appl. 2, 117-173 (1993)

25. Pedlosky, J.: Geophysical Fluid Dynamics. Springer, New York (1987)

26. Rabinowitz, P.H.: Existence and nonuniqueness of rectangular solutions of the Bénard problem. Arch. Ration. Mech. Anal. 29, 179-235 (1968)

27. $\mathrm{Xu}, \mathrm{F}$ : Regularity criterion of weak solution for the $3 \mathrm{D}$ magneto-micropolar fluid equations in Besov space. Commun. Nonlinear Sci. Numer. Simul. 17, 2426-2433 (2012)

28. Zhang, Z: A remark on the blow-up criterion for the 3D Hall-MHD system in Besov spaces. J. Math. Anal. Appl. 441, 692-701 (2016) 